\title{
Colliding pulse mode locking of quantum cascade lasers
}

\author{
Jesus Humberto Abundis-Patino*, Michael Riesch, Petar Tzenov, and Christian Jirauschek \\ Technical University of Munich, D-80333 Munich, Germany
}

\begin{abstract}
We study the possibility for ultrashort pulse generation from $\mathrm{THz}$ quantum cascade lasers via the colliding pulse mode locking technique. Our analysis shows that this approach could enable sub-ps pulses from QCLs, even in devices with carrier recovery times as short as $\sim 10$ ps.
\end{abstract}

\section{Introduction}

Despite being the most potent and compact direct sources of $\mathrm{THz}$ light, due to their dynamic complexity and fast carrier relaxation times, quantum cascade lasers (QCLs) have proven to be difficult to mode lock. The shortest pulses generated to date are on the order of $\sim 2.5 \mathrm{ps}$, and they were produced via an intricate combination between active modulation of the applied bias and injection-seeding the laser [1]. On the other hand, passive mode locking has not been demonstrated to date, as it is widely believed that the fast gain recovery time would naturally inhibit the application of this standard technique.

As already mentioned, QCLs exhibit very peculiar dynamics, where the complex interplay between fast carrier relaxation, giant nonlinearities and spatial hole burning determines the emission characteristics of these devices. QCLs are broadband lasers, with the onset of multimode emission being very close to lasing threshold. Interestingly, recent studies indicate that the light emitted from free running QC lasers, is both frequency and amplitude modulated, with the latter modulation occurring at very short time-scales [2].

In this work we will show that one can exploit this natural "multi-pulse" regime of operation to achieve a very stable second harmonic generation via the well-known colliding pulse mode locking (CPML) technique [3]. In traditional CPML, a fast saturable absorber (FSA) is sandwiched between two equally long active media, eventually leading to the formation of two identical pulses, symmetrically distributed around the geometrical centre of the cavity, which collide inside the FSA. From the point of view of an active cell inside the cavity, the presence of two identical pulses reduces the effective round trip time by a factor of two, which in the case of QCLs could enable their passive mode locking. Importantly, such higher harmonic mode locked devices could be used as stable highrepetition rate local oscillators for various heterodyne measurement applications [4].

\footnotetext{
*Corresponding author: jesus.abundis@tum.de
} 


\section{Model}

The cavity geometry envisaged by us is illustrated in Fig. 1. The device consists of two gain media of equal length, constructed from a suitably engineered, slow gain recovery, bound to continuum QCL [5]. The fast saturable absorber is assumed to consist of the same quantum well heterostructure as the gain, however it is electrically isolated from the former and is biased at an operating point where it acts as a detector rather than an emitter. When certain conditions are met, i.e. fast recovery of the absorber inversion lifetime as well as ultra-strong coupling of the FSA to the optical field, generation of a pair of colliding pulses becomes possible.

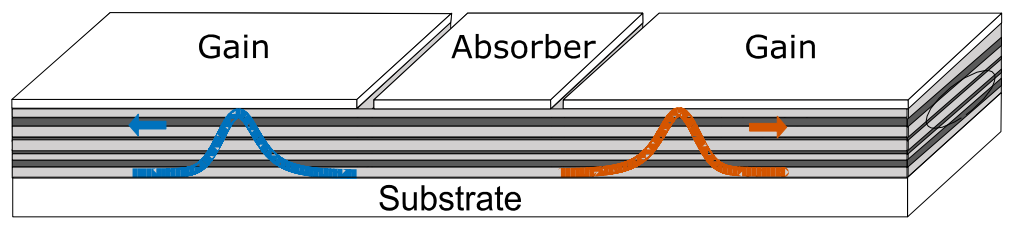

Fig. 1. (a) An example of multi-section Fabry-Perot (FP) cavity geometry, consisting of spatially separated gain $(\mathrm{G})$ and absorber $(\mathrm{A})$ regions for colliding pulse mode locking.

To verify our approach, we employ semi-classical models for both the gain and the absorber, which are based on the solution of the full Maxwell-Bloch equations [6]. Compared to the traditionally used "travelling wave" models, which treat the carrier dynamics via classical rate equations, our approach resolves the electromagnetic field in space and time, solves the quantum mechanical von Neumann equation to evaluate the light-matter interaction dynamics, while it also includes important effects known to play a role in quantum cascade lasers, such as dispersion, optical nonlinearities and spatial hole burning. Notably, we do not employ the commonly used rotating wave approximation which fails for large spectral pulse widths. Furthermore, with the goal for transparency and reproducibility of our results by experimental and theoretical groups alike, we have released our simulation framework as an open source program for the community to exploit $[6,7]$.

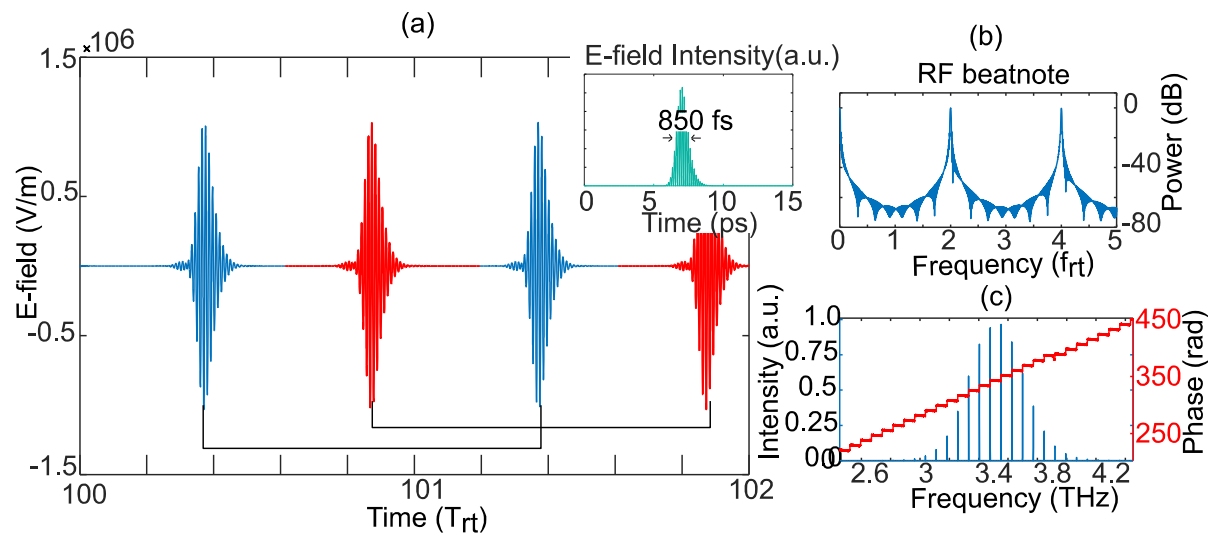

Fig. 2. (a) Time domain profile of the electric field inside the cavity, over time duration of two round trip times (Trt). (Inset) electric field intensity versus time of a single pulse. (b) A log plot of the beatnote signal versus round-trip frequency frt. (c) Optical spectrum and modal phase of the field emitted from the right facet of the cavity.

Figure 2(a)-(c) illustrates the results from our simulations, started from random $\delta$ correlated noise for the electric field, with values for the gain and absorber parameters we believe realistic for QCLs [5]. Our calculations indicate that for gain recovery times $(\sim 10$ 
ps) as fast as 3 times shorter than the cavity round trip time, CPML is possible and above all, is robust against variations of the experimental parameters. Figure 2(a) illustrates the time-trace of the electric field from one such simulation. We see that after 100 round trips of the light inside the cavity, the field splits into two identical pulses per round trip, each with intensity full width at half maximum of approximately 850 fs. Beatnote and optical spectrum calculations also confirm the second harmonic character of the mode locking, Fig. 2(b) and (c), whereas the calculated phases indicate that a fixed phase relationship is established between all lasing Fabry-Perot modes. Additional simulations show that this mechanism is maintained over variations of the cavity length and the injection current, and thus can be seen to provide a stable alternative to active mode locking.

\section{Conclusion}

We propose the usage of CPML for the generation of few cycle terahertz pulses from QCLs. This relatively well known technique can be re-engineered and successfully employed in the development of multi-section quantum cascade laser designs for ultrashort pulse generation. Via rigorous simulations of an extended version of the Maxwell-Bloch system of equations, without invoking the common rotating wave approximation, we demonstrate the validity and robustness of this approach. We investigate the dependence of this second harmonic mode locking onto the cavity length, asymmetries in the geometry, as well as variation of the injection current and other experimental parameters. All of our results indicate that CPML is a promising alternative to active mode locking or conventional passive mode locking as it relaxes the stringent requirement onto the gain recovery versus cavity round trip times, which is usually the limiting factor for pulse generation in QCLs due to their ultrafast dynamics. Additionally, we provide the scientific and engineering communities with an open-source solver for these complex set of equations, which is heavily optimized for performance and could serve for the computer aided design and optimization of future active regions.

\section{References}

1. D. Bachmann, M. Rösch, M. Süess, M. Beck, K. Unterrainer, D. Juraj, J. Faist, G. Scalari, Optica 3, 1087 (2016)

2. I.-C. Benea-Chelmus, M. Rösch, G. Scalari, M. Beck, J. Faist, Phys. Rev. A 96, 033821 (2017)

3. Y.K. Chen, M.C. Wu, IEEE J. Quantum Electron. 28, 2176 (1992)

4. G. Sonnabend, D. Wirtz, R. Schieder, Appl. Opt. 44, 7170 (2005)

5. P. Tzenov, I. Babushkin, R. Arkhipov, M. Arkhipov, N. Rosanov, U. Morgner, C. Jirauschek, New J. Phys. 20, 053055 (2018)

6. M. Riesch, N. Tchipev, S. Senninger, H.-J. Bungartz, C. Jirauschek, Opt. Quant. Electron. 50, 112 (2018)

7. “mbsolve" project, https://github.com/mriesch-tum/mbsolve 